\title{
On the Relationship between Spatial Logics and Behavioral Simulations ${ }^{\star}$
}

\author{
Lucia Acciai $^{1}$, Michele Boreale ${ }^{1}$, and Gianluigi Zavattaro ${ }^{2}$ \\ ${ }^{1}$ Dipartimento di Sistemi e Informatica, Università di Firenze, Italy \\ ${ }^{2}$ Dipartimento di Scienze dell'Informazione, Università di Bologna, Italy
}

\begin{abstract}
Spatial logics have been introduced to reason about distributed computation in models for concurrency. We first define a spatial logic for a general class of infinite-state transition systems, the Spatial Transition Systems (STs), where a monoidal structure on states accounts for the spatial dimension. We then show that the model checking problem for this logic is undecidable already when interpreted over Petri nets. Next, building on work by Finkel and Schnöbelen, we introduce a subclass of sTs, the Well-Structured sTs (ws-sTs), which is general enough to include such models as Petri nets, Broadcast Protocols, ccs and Weighted Automata. Over ws-sTs, an interesting fragment of spatial logic - the monotone fragment - turns out to be decidable under reasonable effectiveness assumptions. For this class of systems, we also offer a Hennessy-Milner theorem, characterizing the logical preorder induced by the monotone fragment as the largest spatial-behavioural simulation. We finally prove that, differently from the corresponding logic, this preorder is in general not decidable, even when confining to effective ws-sTs.
\end{abstract}

\section{Introduction}

Spatial logics [6, 7] are modal logics for describing the behavior and spatial structure of concurrent systems. Beside propositional and temporal operators, they include spatial operators, the most prominent of which is _ $\left.\right|_{-}$, having the following meaning: the formula $\phi_{1} \mid \phi_{2}$ is satisfied by any process that can be decomposed into two processes that satisfy respectively $\phi_{1}$ and $\phi_{2}$. Spatial logics have been applied to several models, such as the pi-calculus [7] and the Ambient Calculus [9].

Starting from the well-known correspondence between the Hennessy-Milner logic and bisimulation [19], a rich literature has been dedicated to the study of the relationship between modal logics and behavioural equivalences or preorders. In the realm of spatial logics, this study has been undertaken in [20, 24], in the case of the Ambient Calculus, and in [5, 8], in the case of the ccs and pi-calculus. A discussion on these works, which are strongly related to our study, is deferred to the concluding section. The objective of the present paper is to start such an investigation in a general setting. To this aim, we introduce the notion of spatial transition system (STs): a possibly infinite-state transition system, endowed with a monoidal structure on states representing the spatial

\footnotetext{
^ Research partly supported by the EU within the FET-GC2 initiative, project SENSorIa. The third author is partially supported by the EU integrated project HATS.
} 
dimension. We introduce a very simple spatial logic $\mathcal{L}$, consisting of atomic predicates, the and/or/not logical operators, a behavioral modality indicating the possibility to reach a state with a given property, and the spatial operator described above. We then interpret $\mathcal{L}$ over sts's, relating the spatial operator to the monoidal structure. On the one hand, STs are general enough to include models such as the Calculus of Communicating Systems (ccs, [22]), Petri nets (and variants thereof, such as reset nets, transfer nets and broadcast protocols), and weighted automata. On the other hand, even if $\mathcal{L}$ is very simple, it is enough expressive to describe interesting properties, such as the impossibility for a ccs process to reach a state where a race condition on a channel arises, or one-safety on Petri nets (i.e. all places in all reachable markings contain at most one token).

Our first result is that model checking of $\mathcal{L}$ is indeed in general undecidable in infinite state systems, even for quite simple sts like Petri nets. This leads us to considering the negation-free fragment of $\mathcal{L}$, written $\mathcal{L}_{0}$ and introduce the class of Well-Structured Spatial Transition Systems (ws-sTs). The latter builds on the class of Well-Structured Transition Systems introduced by Finkel and Schnöbelen [17]; in particular, the existence of a well-quasi order on states that is compatible with both the transition and the monoidal structure of the system plays a crucial role. The class of ws-sTs is still general enough to include all the models mentioned above. We prove that $\mathcal{L}_{0}$ is decidable for the class of ws-STs, subject to some reasonable effectiveness conditions.

Next, we characterize the logical preorder induced by $\mathcal{L}_{0}$, that is, the preorder that relates $s$ to $t$ whenever $t$ satisfies all the $\mathcal{L}_{0}$-formulae satisfied by $s$. We present a coinductive characterization of the logical preorder in terms of a (weak) simulation, enriched with constraints on the spatial properties of $s$ and $t$ and the basic predicates they satisfy (a Hennessy-Milner theorem for $\mathcal{L}_{0}$ ). This simulation, that we call spatial-behavioral preorder (sBs), is, in fact, the largest well-quasi order that is compatible with the spatial and transition structure of the system.

Finally, we show that, differently from $\mathcal{L}_{0}$, this preorder is in general not decidable, even restricting to effective ws-sTs.

Structure of the paper. In Section 2 we define sTs and sBS and introduce some concrete instances that will be used throughout the paper. In Section 3 we introduce $\mathcal{L}$ and its fragment $\mathcal{L}_{0}$, the considered spatial logics, and we prove the undecidability of $\mathcal{L}$ in Petri nets and ccs. Section 4 , after introducing some background material and defining the class of effective ws-sTs, discusses the decidability of $\mathcal{L}_{0}$. In Section 5 we prove that the preorder induced by $\mathcal{L}_{0}$ coincides with the largest sBs, and in Section 6 we prove that the latter is not decidable in ws-sTs in general. A few remarks on further and related work conclude the paper in Section 7. Due to lack of space, some proofs have been left out of this short version (they can be found in [1]).

\section{Spatial Transition Systems}

In this section we introduce spatial transition systems, we provide some instances that will be used as running examples throughout the paper, and we introduce a natural extension of weak simulation for spatial transition systems. 


\subsection{Basic Definitions}

Recall that a transition system $(\mathrm{Ts})$ is a structure $(S, \rightarrow)$ where $S$, ranged over $s, t, \ldots$, is a set of states and $\rightarrow \subseteq S \times S$ is a set of transitions. We let $\rightarrow^{*}$ be the reflexive and transitive closure of $\rightarrow$. The set of immediate predecessors and predecessors of a state $s \in S$ are defined respectively as $\operatorname{Pred}(s)=\{t \mid t \rightarrow s, t \in S\}$ and $\operatorname{Pred}^{*}(s)=\left\{t \mid t \rightarrow{ }^{*} s, t \in S\right\}$. The definitions of Pred and Pred* are extended to sets of states as expected. In the following, we let At be a finite set of atomic predicates ranged over $\mathrm{p}, \mathrm{p}^{\prime}, \ldots$.. We let $\mathcal{P}\left(\right.$ At) denote the powerset of At. Recall that a monoid $\left(M, \oplus, 0_{M}\right)$ is a semigroup with $0_{M}$ as an identity element.

Definition 1 (spatial transition system). A spatial transition system (sTs) is a tuple $\mathcal{S}=(S, \rightarrow, \oplus, \mathrm{O})$ where: $(1)(S, \rightarrow)$ is a transition system, (2) $\left(S, \oplus, 0_{S}\right)$ is a monoid for some $0_{S} \in S$, and (3) $\mathrm{O}: S \rightarrow \mathcal{P}(\mathrm{At})$ is an observation function.

The relationship among the transition system, the monoid and the observation function is given by the following spatial-behavioral simulation.

Definition 2 (spatial-behavioral simulation). A spatial-behavioral simulation (sBs) over a sTs $\mathcal{S}=(S, \rightarrow, \oplus, \mathrm{O})$ is a binary relation $\mathcal{R} \subseteq S \times S$ such that whenever $s \mathcal{R} t$ then:

1. whenever $s \rightarrow s^{\prime}$ then there exists $t^{\prime} \in S$ such that $t \rightarrow^{*} t^{\prime}$ and $s^{\prime} \mathcal{R} t^{\prime}$;

2. whenever $s=s_{1} \oplus s_{2}$ then there are $t_{1}, t_{2} \in S$ such that $t=t_{1} \oplus t_{2}$ and $s_{i} \mathcal{R} t_{i}, i=1,2$;

3. $\mathrm{O}(s) \subseteq \mathrm{O}(t)$.

The largest SBS, denoted $\sqsubseteq$, is a preorder over $S$, called spatial-behavioral preorder.

\subsection{Concrete Instances of STS}

Calculus of Communicating Systems. The fragment of ccs with input guarded replication 1 instead of recursion can be turned into a STs as detailed in [4], that is, working modulo structural congruence and identifying $\oplus$ and its identity with parallel composition | and 0, respectively. Moreover, the relation $\leq$ introduced in Definition 14 of [4] can be easily proved to be a spatial-behavioral simulation w.r.t. this STs.

Affine Well-Structured Nets. We consider a generalization of Petri nets (PN) introduced by Finkel et al.: as discussed in Section 7.2 of [16], PN, Double PN, Generalized Transfer PN (thus also Broadcast Protocols [12]) and Reset PN are all instances of affine wsN. Let $\mathbb{N}^{p}$, for $p \geq 1$, be ranged over by $n, m, \ldots$. Let $m(i)$ denote the $i^{\text {th }}$ component of $m$. The preorder $\leq \subseteq \mathbb{N}^{p} \times \mathbb{N}^{p}$ is defined point-wise as expected: $n \leq m$ if and only if for each $i=1, \ldots, p, n(i) \leq m(i)$. A well-structured net (wsN) is a triple $N=\left(\mathbb{N}^{p}, F, \preceq\right)$, where $\mathbb{N}^{p}$ is the set of states (in the PN terminology, markings on the places $1, \ldots, p$ ) and $F$ is a finite set of partial functions $f, f^{\prime}, \ldots: \mathbb{N}^{p} \rightarrow \mathbb{N}^{p}$, whose domain is an upward-closed subset of $\mathbb{N}^{p}$ - that is, whenever $m \leq n$ and $m \in \operatorname{dom}(f)$ then $n \in \operatorname{dom}(f)$, for each $f \in F$. An affine wSN is a WSN where for each function $f \in F$ there is a square matrix $A \in \mathbb{N}^{p \times p}$

\footnotetext{
${ }^{1}$ Intuitively, the replicated process !a.P corresponds to an infinite number of copies of $a . P$ in parallel: $a . P|\cdots| a . P \mid \cdots$.
} 
and a vector $B \in \mathbb{Z}^{p}$ such that for each $m \in \operatorname{dom}(f), f(m)=A \cdot m+B$ with $m, B$ seen as column vectors. Let us fix an affine wsN $N$. Clearly, $\left(\mathbb{N}^{p},+, 0^{p}\right)$, with + indicating sum of vectors, is a monoid. Define At to be $\{1, \ldots, p\}$ and, for any $n \in \mathbb{N}^{p}, \mathrm{O}(n)=\{i \mid n(i) \neq 0\}$. Finally, define the transition relation: $n \rightarrow_{F} m$ iff $f(n)=m$ for some $f \in F$ (see [16]). Clearly, ( $\left.\mathbb{N}^{p}, \rightarrow_{F},+, \mathrm{O}\right)$ is a sts for which $\leq$ is a sBs (due to the fact that each $f \in F$ is monotone). Moreover, $\leq$ is also the spatial-behavioral preorder (i.e. the largest SBs).

There are interesting variations of this construction, corresponding to choosing different observation functions $O$. For instance, one can leave the set At unspecified, fix a labelling function from places to sets of atomic predicates, $l:\{1, \ldots, p\} \rightarrow \mathcal{P}(\mathrm{At})$, and then let $\mathrm{O}(m)=\bigcup_{i \in 1 . . p: m(i)>0} l(i)$. Yet another possibility is observing enabled transitions in the current state: assuming $F=\left\{f_{i} \mid i \in I\right\}$, this is obtained by letting At $=I$ and $\mathrm{O}(m)=\left\{i \in I \mid m \in \operatorname{dom}\left(f_{i}\right)\right\}$. These variations too give rise to sTs for which $\leq$ is a sBs, but in general not the largest one.

Weighted Automata. Recall that a semiring $\mathbb{K}$ is a structure $(K,+, \times, 0,1)$ such that $(K,+, 0)$ is a commutative monoid, $(K, \times, 1)$ is a (not necessarily commutative) monoid, $\times$ distributes over + both to the left and to the right and 0 annihilates both to the left and to the right (i.e., $0 \times a=a \times 0=0$ for each $a \in K$ ). Now let us fix a semiring $\mathbb{K}$ and consider the following preorder over $K: a \leq b$ iff there is $c \in K$ s.t. $a+c=b$. With these definitions, the construction illustrated above for Affine Nets carries over formally unchanged when replacing both $\mathbb{N}$ and $\mathbb{Z}$ by $\mathbb{K}$. Doing so, we cast (a generalization of) Weighted Automata (wA, [21]) into the framework of st:2. Concrete instances are: $\mathbb{K}=\mathbb{N}, \mathbb{K}=\mathbb{Q}^{+}$(positive rationals) and $\mathbb{K}=\mathbb{R}^{+}$(positive reals, hence e.g. finite-state Markov chains). Another instance is the (max, +) (a.k.a. tropical semiring [25]) used in quantitative evaluation of discrete-time systems [3]. The latter is defined over $\mathbb{N} \cup\{\infty\}$, by letting "+" to be max and " $x$ " to be + .

\section{The Logics $\mathcal{L}$ and $\mathcal{L}_{0}$}

\subsection{Definitions and Examples}

Definition 3. The set $\mathcal{L}$ of logic formulae $\phi, \psi, \ldots$ is given by the following syntax, where $\mathrm{p} \in$ At: $\phi::=\mathrm{p}|\phi| \phi\left|\diamond^{*} \phi\right| \neg \phi|\phi \wedge \phi| \phi \vee \phi$.

The set of logical operators includes spatial modalities (atomic predicates $\mathrm{p} \in \mathrm{At}$, the composition operator "|"), dynamic connectives (the eventuality modality $\diamond^{*}$ ), and the usual boolean connectives $(\neg, \wedge, \vee)$. The interpretation of $\mathcal{L}$ over a sTs is given below.

$$
\begin{gathered}
\llbracket \mathrm{p} \rrbracket=\{s \in S \mid \mathrm{p} \in \mathrm{O}(s)\} \quad \llbracket\left[\diamond^{*} \phi \rrbracket=\operatorname{Pred}^{*}(\llbracket \phi \rrbracket) \quad \llbracket \neg \phi \rrbracket=S \backslash \llbracket \phi \rrbracket\right] \\
\llbracket \phi_{1} \vee \phi_{2} \rrbracket=\llbracket\left[\phi _ { 1 } \rrbracket \cup \llbracket \left[\phi _ { 2 } \rrbracket \quad \llbracket \left[\phi_{1} \wedge \phi_{2} \rrbracket=\llbracket\left[\phi _ { 1 } \rrbracket \cap \llbracket \left[\phi_{2} \rrbracket\right.\right.\right.\right.\right. \\
\llbracket\left[\phi_{1} \mid \phi_{2} \rrbracket=\left\{s_{1} \oplus s_{2} \mid s_{j} \in \llbracket\left[\phi_{j} \rrbracket \text { for } j=1,2\right\}\right.\right.
\end{gathered}
$$

Connectives are interpreted as expected. In particular, satisfiability of the basic predicates is given by $\mathrm{O}$ while satisfiability of the composition operator relies on the possibility of decomposing states via $\oplus$. In what follows we usually write $s \vDash \phi$ if $s \in[[\phi]$.

\footnotetext{
${ }^{2}$ Concretely, states $s, t, \ldots$ of the wA are elements of $\mathbb{K}^{p \times 1}$, and $s \rightarrow t$ iff $t=A \cdot s$, where $A \in \mathbb{K}^{p \times p}$
} is the transition matrix of the wa. 
Following [2], we say that a formula is monotone if it does not contain any occurrence of $\neg$. We let $\mathcal{L}_{0}$ denote the subset of monotone formulae. A formula is anti-monotone if it is of the form $\neg \phi$ with $\phi$ monotone.

Example 1. The following formulae for ccs depends on generic names $a$ and $b$. The anti-monotone formula $\phi=\neg \diamond^{*}(\bar{a} \wedge \bar{b})$ says that the output on $a$ and $b$ are forever mutually exclusive; e.g. $\bar{a}+\bar{b} \not \models \phi$. This is different from $\psi=\neg \diamond^{*}(\bar{a} \mid \bar{b})$, still anti-monotone, saying that it will never be the case that there are two independent threads in the system offering an output on $a$ and one on $b$; e.g. $\bar{a}+\bar{b} \models \psi$, while $\bar{a} \mid \bar{b} \not \neq \psi$.

Similar properties can be defined in the case of wsN. Let $i, j \in\{1, \ldots, p\}$. The formula $\neg \diamond^{*}(i \wedge j)$ says that no marking is reachable where place $i$ and place $j$ are non-empty. This is equivalent to $\neg \diamond^{*}(i \mid j)$ if $i \neq j$, while $\neg \diamond^{*} \bigvee_{l=1}^{p}(l \mid l)$ says that the net is onesafe. Switching the alternative interpretation where enabled transitions are observed, the formula $\neg \diamond^{*}(i \wedge j)$ would say that no marking is reachable where both $f_{i}$ and $f_{j}$ are enabled. This is now stronger than $\neg \diamond^{*}(i \mid j)$, saying that no marking is reachable where $f_{i}$ and $f_{j}$ can both fire simultaneously. The logic can also be used to define properties that go beyond pure coverability, such as $\left(\diamond^{*} a\right) \mid\left(\diamond^{*} b\right)$, saying that, from the current state, two non conflicting transitions on $a$ and $b$ can be reached. These formulae also make sense in the case of wA. As an example, in a Markov chain, $\neg \diamond^{*}(i \mid j)$ says that it is not possible to reach a state where both transition $i$ and transition $j$ have non-zero probability.

\subsection{Undecidability of $\mathcal{L}$}

We now prove that the logic $\mathcal{L}$ turns out to be undecidable already for Petri nets PN, one of the simplest instantiations of affine wsN in which the matrix $A$, used in the definitions of the functions $f$, always corresponds with the identity matrix.

The proof is by reduction from the containment problem for PN. An instance of the containment problem consists of two PN $\Sigma_{1}, \Sigma_{2}$ with the same number of places, and a bijection $g$ between the sets of places of $\Sigma_{1}$ and $\Sigma_{2}$ ( $g$, called renaming in the following, is extended to a bijection between markings in the obvious way). The problem consists of checking whether for every reachable marking $m$ of $\Sigma_{1}, g(m)$ is reachable in $\Sigma_{2}$. Rabin showed that this problem is undecidable (the proof is in [18]). Following the approach used in [13] to prove the undecidability of the modal $\mu$-calculus for PN, we reduce the containment problem to the problem of model checking a given formula in a PN.

Assume that the number of places of $\Sigma_{1}$ and $\Sigma_{2}$ is $p$. We now define a PN $\left.\left[\Sigma_{1}, \Sigma_{2}\right]\right]=$ $\left(\mathbb{N}^{2 p+4}, F, \leq\right)$ where $\leq$ is the usual ordering on naturals extended to vectors. The states of $\left[\left[\Sigma_{1}, \Sigma_{2}\right]\right.$ are vectors of length $2 p+4$ : the first $p$ elements are used to represent states of $\Sigma_{1}$, the subsequent $p$ elements are used to represent states of $\Sigma_{2}$, while the last 4 elements are used to divide the computations in four distinct phases. The first phase is a simulation of a computation of $\Sigma_{1}$, the second phase is the passage through a specific observable state, the third phase is a simulation of $\Sigma_{2}$, and the last phase is used to check whether the markings reached by the simulations of $\Sigma_{1}$ and $\Sigma_{2}$ correspond up-to renaming.

Formally, the computations of $\left[\left[\Sigma_{1}, \Sigma_{2}\right]\right]$ are controlled by $F$ containing the three classes of functions defined below, where we use $x \cdot y$ to denote the juxtapositions of the vectors $x$ and $y$ : 
- $F$ contains the functions $f_{1}(x)=x+0^{2 p} \cdot(-1,1,0,0), f_{2}(x)=x+0^{2 p} \cdot(0,-1,1,0)$, and $f_{3}(x)=x+0^{2 p} \cdot(0,0,-1,1)$;

- for each $f(x)=x+B$ in $\Sigma_{1}$ (resp. $\left.\Sigma_{2}\right), F$ contains a corresponding $f(x)=x+B \cdot 0^{p+4}$ (resp. $\left.f(x)=x+0^{p} \cdot B \cdot 0^{4}\right)$ defined only if $x(2 p+1)>0$ (resp. if $x(2 p+3)>0$ );

- for each place $s$ in $\{1, \cdots, p\}, F$ contains a function $f(x)=x+B_{s}$, defined only if $x(2 p+4)>0$, where $B_{s}$ contains -1 in the positions $s$ and $p+g(s)$, and 0 elsewhere.

Assuming that the initial states of $\Sigma_{1}$ and $\Sigma_{2}$ are respectively $m_{1}$ and $m_{2}$, we will consider for $\llbracket\left[\Sigma_{1}, \Sigma_{2}\right]$ the state $m_{1} \cdot m_{2} \cdot(1,0,0,0)$.

We now discuss the meaning of the three classes of functions defined above. The first three functions dictate the passage from one phase to the subsequent one. In the second class of functions, those of the form $f(x)=x+B \cdot 0^{p+4}$ (resp. $f(x)=x+0^{p} \cdot B \cdot 0^{4}$ ) are used to mimic the computations of $\Sigma_{1}$ (resp. $\Sigma_{2}$ ) during the first (resp. the third) phase. The third class of functions reduces in a synchronized manner the values in the places $s$ and $p+g(s)$. In this way, if a state with all the first $2 p$ elements equal to 0 is reached, we can conclude that the markings reached during the simulations of the computations of $\Sigma_{1}$ and $\Sigma_{2}$ correspond up-to renaming. We now prove a proposition that formalizes the correctness of the reduction: in the statement of the proposition we exploit the fact that every state $x$ reachable in $\llbracket \Sigma_{1}, \Sigma_{2} \rrbracket$ such that $x(2 p+2)>0$ is an intermediary state between the simulation of a computation of $\Sigma_{1}$ (performed while the element in position $2 p+1$ is 1 ) and the subsequent simulation of a computation of $\Sigma_{2}$ (performed while the element in position $2 p+3$ is 1 ).

Proposition 1. Given two Petri nets $\Sigma_{1}, \Sigma_{2}$ with initial markings $m_{1}$ and $m_{2}$, respectively, we have that they satisfy the containment problem iff for every computation of $\llbracket\left[\Sigma_{1}, \Sigma_{2}\right]$ starting from $m_{1} \cdot m_{2} \cdot(1,0,0,0)$ and leading to a state $x$ such that $x(2 p+2)>0$, then the computation can be extended in order to reach a state in which the first $2 p \mathrm{el}$ ements are all equal to 0 .

In the light of this theorem we conclude that two Petri nets $\Sigma_{1}$ and $\Sigma_{2}$, with initial markings $m_{1}$ and $m_{2}$, satisfy the containment problem iff for the PN $\left[\llbracket \Sigma_{1}, \Sigma_{2}\right]$ we have

$$
m_{1} \cdot m_{2} \cdot(1,0,0,0) \vDash \neg \diamond^{*} \neg\left(\neg(2 p+2) \vee\left(\diamond^{*} \bigwedge_{i \in\{1, \cdots, 2 p\}} \neg i\right)\right)
$$

from which we get the following result.

Theorem 1. The model checking of $\mathcal{L}$ is undecidable for $\mathrm{PN}$.

It is worth noting that the logic $\mathcal{L}$ is undecidable also for the fragment of ccs introduced in Section 2.2. This can be proved as a corollary of an undecidability result in [4]. In that paper (see Section 3) weak bisimulation is proved to be undecidable, for this fragment of ccs, presenting a nondeterministic modeling of Random Access Machines (RAMs) [23], a well known register based Turing complete formalism. The encoding is nondeterministic because it can give rise to computations that do not correspond to the computation of the modeled RAM. Nevertheless, those computations generate subprocesses that performs infinite computations presenting the barb $w^{\prime}$ infinitely often. So we have that a RAM $R$ terminates iff the corresponding encoding $[[R]]$ in $\operatorname{ccs}$ has a computation leading to a halt instruction - in the encoding in [4] halt instructions present the barb $\bar{w}$ - but without subprocesses left by wrong computations, that is, iff $\llbracket R] \models \diamond^{*}\left(\bar{w} \wedge\left(\neg \diamond^{*} w^{\prime}\right)\right)$. 


\section{Decidability of $\mathcal{L}_{0}$}

In this section we show the existence of a significant sub-class of Spatial Transition Systems, that we call effective Well-Structured Spatial Transition Systems (ws-STs), for which the monotone fragment $\mathcal{L}_{0}$ turns out to be decidable. Basically, ws-sTs enhance classical wsTs [17] by taking into account the spatial structure given by the monoid $(S, \oplus, 0)$.

\subsection{Well-Structured Spatial Transition Systems}

Recall that a quasi-ordering (Qo) (aka preorder) over $S$ is a reflexive and transitive relation over $S$. A well-quasi-ordering (WQo) is a Qo $\leq$ over $S$ such that for any infinite sequence $s_{1}, s_{2}, \ldots$ in $S$ there exists indexes $i<j$ such that $s_{i} \leq s_{j}$; in other words, $S$ does not have infinite antichains. For any Qo $\leq$ over $S$ and $T \subseteq S$, we say $s \in T$ is a minimal element of $T$ if for each $t \in T, s \leq t$; we let $\operatorname{Min}(T)$ denote the set of minimal elements of $T$. A well-structured spatial transition system is a sTs equipped with a QO that is compatible with $\rightarrow, \oplus$ and $\mathrm{O}$.

Definition 4 (well-structured spatial transition system). $A$ well-structured spatial transition system (ws-STS) is a sTS $\mathcal{S}=(S, \rightarrow, \oplus, \mathrm{O})$ equipped with a wQo $\leq$ over $S$ satisfying the following conditions: (1) $\leq$ is a SBS, (2) whenever $s \leq s^{\prime}$ then for each $t s \oplus t \leq s^{\prime} \oplus t$ and $t \oplus s \leq t \oplus s^{\prime}$, and (3) $0_{S} \in \operatorname{Min}(S)$.

Example 2. The concrete instances of sts provided in Section 2.2 are ws-sTs. Theorem 6 of [4] proves that $\leq$ is a WQo over ccs, while clauses (1), (2) and (3) can be easily checked.For what concerns affine wsN, clearly $\leq$ is a wQo because it is defined as the pointwise extension of $\leq$ over $\mathbb{N}$, which is a wQo (Dickson's Lemma [10]). Clause (1) of Definition 4 has been discussed in Section 2.2 and clauses (2) and (3) can be easily checked. Hence, $\left(\mathbb{N}^{p}, F,+, \mathrm{O}\right)$ is a ws-sTs for any variation of $\mathrm{O}$. Essentially the same reasoning applies to weighted automata. Concrete instances where $\leq$ is a wQo, hence the construction yields a ws-sTs, are $\mathbb{K}=\mathbb{N}, \mathbb{K}=\mathbb{R}^{+}$and $\mathbb{K}=(\max ,+)$.

\subsection{Decidability of $\mathcal{L}_{0}$ for Effective ws-STS}

Before presenting the technical machinery needed to define effective ws-sTs and to prove the decidability of $\mathcal{L}_{0}$ for this particular class of ws-sTs, we present the following lemma stating that $\models$ on monotone formulae is compatible with both sBs and $\oplus$.

Lemma 1. Let $(S, \rightarrow, \oplus, \mathrm{O})$ be a ws-sTs and $s, t \in S$. Let $\phi$ be a monotone formula. (1) If $s \sqsubseteq t$ and $s \models \phi$ then $t \models \phi$. (2) If $s \models \phi$ then, for each $t$ also $s \oplus t \models \phi$.

Let us introduce some auxiliary notations and results first. Given any $s$ in a set $X$ preordered by $\leq$, we let its upward-closure to be $\uparrow s=\{t \mid s \leq t\}$. This notation is extended to any set $I \subseteq X$ as expected. A set $I$ is upward-closed if $\uparrow I=I$. A finite basis of an upwardclosed set $I$ is a finite set $B \subseteq X$ such that $\uparrow B=I$. If $X$ is a wQo, any upward-closed set $I$ has a finite basis. Indeed, it is enough to choose from $\operatorname{Min}(I)$ one representative of each equivalence class induced by $\leq$ : there must be finitely many such classes, otherwise one 
could form an antichain. Now, in any ws-sTs and for any monotone $\phi,[[\phi]]$ is upward closed w.r.t. $\leq($ Lemma 11$)$ ). Hence the existence of a finite basis of $[[\phi]$ is guaranteed. Now we have to show how to build one such basis.

For the rest of the section, let us fix a ws-sts $\mathcal{S}$. We assume three functions $b, \mathrm{pb}^{*}$ and mub, yielding finite bases for certain upward closed sets. Specifically: for each atomic predicate $\mathrm{p}, \mathrm{b}(\mathrm{p})$ yields a finite basis of $[\mathrm{p}]]$, that is $\uparrow \mathrm{b}(\mathrm{p})=[[\mathrm{p}]$; for each finite $I \subseteq S$, $\mathrm{pb}^{*}(I)$ yields a finite basis of $\operatorname{Pred}^{*}(\uparrow I)$; and for each $s_{1}, s_{2} \in S, \operatorname{mub}\left(s_{1}, s_{2}\right)$ yields a finite basis of $\uparrow s_{1} \cap \uparrow s_{2}$, in other words a set of minimal upper bounds for $s_{1}$ and $s_{2}$. For the time being, we make no assumption about the effectiveness of these functions. Building on them, a finite basis of $[[\phi]]$, written $\mathrm{Fb}(\phi)$, is defined below by induction on $\phi$.

$$
\begin{aligned}
& \mathrm{Fb}(\mathrm{p})=\mathrm{b}(\mathrm{p}) \quad \mathrm{Fb}\left(\diamond^{*} \phi\right)=\mathrm{pb}^{*}(\mathrm{Fb}(\phi)) \quad \mathrm{Fb}\left(\phi_{1} \vee \phi_{2}\right)=\mathrm{Fb}\left(\phi_{1}\right) \cup \mathrm{Fb}\left(\phi_{2}\right) \\
& \mathrm{Fb}\left(\phi_{1} \wedge \phi_{2}\right)=\bigcup_{s_{1} \in \mathrm{Fb}\left(\phi_{1}\right), s_{2} \in \mathrm{Fb}\left(\phi_{2}\right)} \operatorname{mub}\left(s_{1}, s_{2}\right) \quad \mathrm{Fb}\left(\phi_{1} \mid \phi_{2}\right)=\bigcup_{s_{1} \in \mathrm{Fb}\left(\phi_{1}\right), s_{2} \in \mathrm{Fb}\left(\phi_{2}\right)}\left\{s_{1} \oplus s_{2}\right\}
\end{aligned}
$$

Proposition 2. Let $\phi$ be monotone. Then $\mathrm{Fb}(\phi)$ is a finite basis of $\llbracket \phi]$.

In order to prove decidability, we need to argue now about effectiveness of b, pb* and mub. In particular, we shall rely on Finkel and Schnöbelen's result below that establishes effectiveness of $\mathrm{pb}^{*}$ under certain conditions. Let us define the pred-basis of a state $s, \mathrm{pb}(s)$, as the finite basis of $\uparrow \operatorname{Pred}(\uparrow s)$ :

$$
\uparrow \operatorname{pb}(s)=\uparrow \operatorname{Pred}(\uparrow s)=\{t \mid t \geq \rightarrow \geq s\} .
$$

Let us say that a ws-sTs has an effective pred-basis if $\mathrm{pb}(\cdot)$ is computable. We say a wsts $\mathcal{S}$ is effective if it has effective pred-basis and $\leq$ is decidable.

Proposition 3 (Proposition 3.5 [17]). If $\mathcal{S}$ is an effective wsts, then it is possible to effectively compute a finite basis of $\operatorname{Pred}^{*}(\uparrow I)$, for any finite $I \subseteq S$. That is, there exists a computable $p b^{*}$ function for $\mathcal{S}$.

We say a ws-sTs $\mathcal{S}$ is effective if it is effective as a wsts and $\oplus, \mathrm{b}(\cdot)$ and $\operatorname{mub}(\cdot, \cdot)$ are computable. By the above proposition and the definition of $\mathrm{Fb}$, we have the following result. The wanted result follows as a corollary.

Proposition 4. Let $\mathcal{S}$ be an effective ws-sts. Then $\mathrm{Fb}(\phi)$ can be effectively computed, for any monotone $\phi$.

Corollary 1 (decidability). Let $\mathcal{S}$ be an effective ws-sTs. For any $s$ and (anti-)monotone $\phi$, it is decidable whether $s \models \phi$.

\subsection{Decidability in Concrete Instances}

Let us discuss effectiveness of the ws-sts introduced in Section 2 In each case, the nontrivial part is actually defining effective pred-basis pb and mub functions. Effectiveness of ccs as a wS-STs can be proved along the lines of [2] (note that the definition of mub turns out to be nontrivial).

Let us now briefly consider affine wsN. Each affine function is recursive, hence effectiveness of the pred-basis follows from Theorem 4.2 of [16]. Next, for any $m, n \in \mathbb{N}^{p}$, 
$\operatorname{mub}(m, n)=l \in \mathbb{N}^{p}$ is defined thus: $l(i)=\max \{n(i), m(i)\}$, for each $i=1, \ldots, p$. Indeed $l$ is computable and $n, m \leq l$. Moreover, by definition, whenever $n \leq k$ and $m \leq k$ then $l \leq k$, hence $\uparrow l=\uparrow n \cap \uparrow m$. These definitions of course apply also to the alternative version of wS-STs with enabled transitions as atomic predicates.

In the case of wA, one must ensure in the first place the effectiveness of the predbasis, that strictly depends on the specific semiring; we leave this problem for future investigations. As an example, the results in [26] seem to indicate that an effective pred-basis exists in the case of tropical semirings.

\section{A Hennessy-Milner Theorem for $\mathcal{L}_{0}$}

In this section we prove that, under certain conditions, the logical preorder induced by the monotone fragment $\mathcal{L}_{0}$ coincides with the largest sBs. The proof goes along the lines of the classical theorem for bisimulation and the Hennessy-Milner logic [22]. However, the proof requires extra care, as it has to work also for non-image-finite processes. Indeed, the condition of image-finiteness, customary in process calculi when dealing with "weak" relations, makes little sense in our setting. When building distinguishing formulas for sBs-unrelated states, this fact will lead us to considering an in general infinite number of derivatives. A similar issue is raised by the monoidal structure of the system. In the end, we will be able to prove the result for a rather general class of ws-STS that enjoy certain monotonicity conditions. In the actual proof, though, we will have to resort to certain continuity arguments in order to cope with the issues outlined above. The technical device to do this is the notion of complete wsts of [14, 15]. Intuitively, in a complete ws-STs, ascending chains of states always converge to limit points, and the limit operation commutes with transitions, sum and observation.

A few preliminary definitions are in order. Let $(X, \preceq)$ be a poset. Recall that a set $D \subseteq X$ is directed if any two elements in $D$ have an upper-bound in $D$. A dcpo is a poset $(X, \preceq)$ where any directed set $D \subseteq X$ has a least upper bound (lub) in $X$, denoted $\vee D$. A dcpo is algebraic if any element $x \in X$ is the lub of the set of finite elements $\leq x$ (recall that $y \in X$ is finite if for every directed $D$, whenever $y \leq \bigvee D$ then $y \leq d$ for some $d \in D)$. The set of finite elements of any poset $X$ is denoted by $\operatorname{fin}(X)$. Let $X, Y$ be two preordered sets. A partial function $f: X \rightarrow Y$ is monotone if $\operatorname{dom}(f)$ is upward closed in $X$ and whenever $x \leq x^{\prime}$ in $\operatorname{dom}(f)$ then $f(x) \leq f\left(x^{\prime}\right)$ in $Y$. When $X$ and $Y$ are depo, we say $f$ is continuous if $f$ is monotone, its domain is Scott-closed (that is, upward-closed, and such that for any directed $D \subseteq X, \vee D \in \operatorname{dom}(f)$ implies $D \cap \operatorname{dom}(f) \neq \emptyset)$ and, for any directed $D, f(\bigvee D)=\bigvee f(D)$. Finally, we say $f$ is finitary if $f(\operatorname{fin}(X)) \subseteq \operatorname{fin}(Y)$.

Following [14], we say a ws-sTs is functional if its transition relation $\rightarrow$ can be decomposed as the union of finitely many transitions functions: $\rightarrow=\cup_{i=1}^{n} \delta_{i}$, where for $i=1, \ldots, n, \delta_{i}: S \rightarrow S$ is a partial monotone function. Note that in a functional wssTs, monotonicity of $\oplus$ and $\mathrm{O}$ (the latter w.r.t. set inclusion in $\mathcal{P}(\mathrm{At})$ ) follows by definition. Complete ws-sTs however require something more than monotonicity. Let us record that for any finite set $I, \mathcal{P}(I)$, partially ordered by set inclusion, is trivially an (algebraic) dcpo.

Definition 5 (complete ws-STs). A complete ws-sTs is a functional ws-sTs $\left(S, \cup_{i=1}^{n} \delta_{i}, \oplus, \mathrm{O}\right)$ such that $(S, \preceq)$ is an algebraic dcpo, each $\delta_{i}: S \rightarrow S$ is a finitary 
continuous partial function and $\oplus: S \times S \rightarrow S$ and $\mathrm{O}: S \rightarrow \mathcal{P}($ At) are finitary continuous total functions.

For each state $s$ in a transition system, define Post* $(s)$ to be the set of states reachable from $s: \operatorname{Post}^{*}(s) \triangleq\left\{s^{\prime} \mid s \rightarrow^{*} s^{\prime}\right\}$. Note that a complete ws-sts in our sense is also a complete wsts in the sense of [14]. Hence we have the following result from [14] about the cover of $s$, that is, the downward closure of Post* $(s)$.

Lemma 2 ([14], Proposition 6.1). In any complete wsts, hence in any complete wsSTs, for any state $s$ there is a finite set $F \subseteq \operatorname{Post}^{*}(s)$ such that $\downarrow \operatorname{Post}^{*}(s)=\downarrow F$.

The following result is a generalization of the previous one to the spatial component.

Lemma 3. Let $\mathcal{S}$ be a complete ws-sTs. For any state $s$, consider the set $D_{s}=\left\{\left(t, t^{\prime}\right) \mid s=\right.$ $\left.t \oplus t^{\prime}\right\}$. Then there is a finite $F \subseteq D_{\text {s }}$ s.t. $\downarrow D_{s}=\downarrow F$ in $S \times S$.

We also need inductively defined approximants of the spatial-behavioral similarity.

Definition 6 (approximants of similarity). Let $\mathcal{S}$ be a ws-sTs. We let $\left(\sqsubseteq_{i}\right)_{i \in \mathbb{N}}$ be the sequence of preorders on $S$ defined by induction on $i$ as follows:

a) $s \sqsubseteq_{0} t$ always;

b) $s \sqsubseteq_{i+1} t$ if: (1) whenever $s \rightarrow s^{\prime}$ then there exists $t^{\prime}$ s.t. $t \rightarrow^{*} t^{\prime}$ and $s^{\prime} \sqsubseteq_{i} t^{\prime}$; (2) whenever $s=s_{1} \oplus s_{2}$ then there exist $t_{1}$, $t_{2}$ s.t. $t=t_{1} \oplus t_{2}$ and $s_{j} \sqsubseteq_{i} t_{j}$ for $j=1,2$; (3) $\mathrm{O}(s) \subseteq \mathrm{O}(t)$.

We let $\sqsubseteq_{\omega}$ be $\cap_{i \in \mathbb{N}} \sqsubseteq_{i}$.

The preorder $\sqsubseteq_{\omega}$ can be proved to coincide with $\sqsubseteq$; the proof relies on arguments similar to those used in the proof of Theorem 2 below.

Proposition 5. On a complete ws-sTs, $\sqsubseteq$ and $\sqsubseteq_{\omega}$ coincide.

Let $\mathcal{S}$ be a spatial transition system. The logical preorder $\sqsubseteq_{\mathcal{L}}$ over states is defined as: $s \sqsubseteq \mathcal{L}_{0} t$ if and only if for each formula $\phi \in \mathcal{L}_{0}, s \models \phi$ implies $t \models \phi$. Here is the first version of the result we are after.

Theorem 2 (Hennessy-Milner type theorem for complete ws-STs). On a complete Ws-STS, $\sqsubseteq$ and $\sqsubseteq \mathcal{L}_{0}$ concide.

Proof: The inclusion $\sqsubseteq \subseteq \sqsubseteq \mathcal{L}_{0}$ holds for any ws-sTs (Lemma 1 (1)). As for the opposite inclusion, it is convenient to work with $\sqsubseteq_{\omega}$ (Proposition 5). The proof then is a variant of the one in [22]. In particular, we prove the contrapositive statement, that $s \nsubseteq \omega t$ implies $s \nsubseteq \mathcal{L} t$. Assume that there is an index $i$ s.t. $s \nsubseteq_{i} t$ : we show the existence of a formula $\phi$ s.t. $s \models \phi$ and $t \not \neq \phi$. The proof is by induction on $i$. Assume $i>0$. Now, $s \nsubseteq_{i} t$ means that one of the clauses (1-3) of Definition 2 is violated.

Let us examine (1) first: there is $s^{\prime}$ s.t. $s \rightarrow s^{\prime}$ and for no $t^{\prime} \in \operatorname{Post}^{*}(t)$ it holds that $s^{\prime} \sqsubseteq_{i-1} t^{\prime}$. Consider the cover of $t, \downarrow$ Post $^{*}(t)=\downarrow F$ for some finite set $F \subseteq$ Post $^{*}(t)$ (Lemma 2). It is not difficult to show that for any $u \in F, s^{\prime} \Phi_{i-1} u$. By induction hypothesis, there exists then $\phi_{u}$ s.t. $s^{\prime} \vDash \phi_{u}$ and $u \not \models \phi_{u}$. Moreover, for each $t^{\prime} \in \downarrow u, t^{\prime} \not \neq \phi_{u}$ either 
(a consequence of Lemma 1). Consider now $\phi=\diamond^{*}\left(\bigwedge_{u \in F} \phi_{u}\right)$. By construction $s \vDash \phi$, but $t \not \neq \phi$.

The case where (2) is violated is handled similarly relying on Lemma 3 In particular, $s=s_{1} \oplus s_{2}$ for some $s_{1}$ and $s_{2}$ s.t. for each pair $\left(t_{1}, t_{2}\right)$ satisfying $t=t_{1} \oplus t_{2}$, either $s_{1} \nsubseteq_{i-1} t_{1}$ or $s_{2} \nsubseteq_{i-1} t_{2}$. Let us write as $\left\{\left(t_{1}^{j}, t_{2}^{j}\right) \mid j \in J\right\}$ ( $J$ finite) the set $F$ given by Lemma 3. By induction, for each $j \in J$ there exists either $\phi_{1}^{j}$ satisfied by $s_{1}$ but not by $t_{1}^{j}$, or $\phi_{2}^{j}$ satisfied by $s_{2}$ but not by $t_{2}^{j}$. In the former case let $\phi_{2}^{j} \triangleq$ true, in the latter case $\phi_{1}^{j} \triangleq$ true. Consider now $\phi=\left(\bigwedge_{j \in J} \phi_{1}^{j}\right) \mid\left(\bigwedge_{j \in J} \phi_{2}^{j}\right)$. By construction, $s \models \phi$, but $t \not \models \phi$. Finally, the case (3) is obvious.

Next, we extend the above result to the class of functional (not necessarily complete) ws-sTs's. Let us introduce some terminology first. For any complete ws-sTs $\mathcal{S}=$ $\left(S, \cup_{i=1}^{n} \delta_{i}, \oplus, \mathrm{O}\right)$, let us denote by $\operatorname{fin}(\mathcal{S})$ the functional wsts $\left(\operatorname{fin}(S), \cup_{i=1}^{n} \delta_{i, \mathrm{fin}}, \oplus_{\mathrm{fin}}, \mathrm{O}_{\mathrm{fin}}\right)$, where fin $(S)$ inherits the wQo of $S$ and $\delta_{i, \mathrm{fin}}, \oplus_{\mathrm{fin}}, \mathrm{O}_{\text {fin }}$ are the restrictions of $\delta_{i}, \oplus$ and $\mathrm{O}$, respectively, to fin $(S)$. Let us denote by $\sqsubseteq_{\text {fin }}$ the spatial-behavioral similarity defined over fin $(\mathcal{S})$ (the subscript fin will be omitted when no ambiguity arises).

Two functional ws-sTs's are isomorphic if there is an embedding between them that is a bijection and commutes with the functions $\delta_{i}(i=1, \ldots, n$, for one and the same $n), \oplus$ and $\mathrm{O}$, as expected. Clearly, any isomorphism preserves, in both directions, both $\sqsubseteq$ and $\vDash$, hence $\sqsubseteq \mathcal{L}_{0}$. Now, given a functional ws-sTs $\mathcal{S}$ there is a canonical way of building a complete ws-sTs $\hat{\mathcal{S}}$ such that $\operatorname{fin}(\hat{\mathcal{S}})$ is isomorphic to $\mathcal{S}$ : one takes the ideal completion of $\mathcal{S}$, where the set of states, $\hat{S}$, is the set of all ideals (that is, directed, downward closed subsets) of $S$ ordered by set inclusion, and $\hat{\delta}_{i}, \hat{\oplus}, \hat{O}$ are the unique continuous extensions of the corresponding (monotone) functions of $\mathcal{S}$. The isomorphism between $\mathcal{S}$ and $\operatorname{fin}(\hat{\mathcal{S}})$ is given by the function $\hat{\bullet}: S \rightarrow \operatorname{fin}(\hat{S})$ that sends each $s \in S$ into $\downarrow s \in \operatorname{fin}(\hat{S})$. A further technical ingredient is needed so as to ensure that $\hat{\mathcal{S}}$ is well-ordered: the wQo $S$ we start with must be a $\omega^{2}$-wqo. Intuitively, $\omega^{2}$-wqo strengthens the condition of wQo in the sense that one can always extract an infinite ascending chain $x_{i, j}<x_{j, k}<x_{k, l}<\cdots$, with $i<j<k<l$, out of any family of elements $\left\{x_{m, n}\right\}_{m \in I, n \in J}$. We refer the reader to [14] for the formal definition of this concept and further details of this construction; here, we just stress that only pathological instances of non- $\omega^{2}$-wqo exist, and they have no computational relevance. With these definitions and facts at hand, the main result of the section is an easy consequence of Theorem 2

Theorem 3 (Hennessy-Milner type theorem, functional case). Let $\mathcal{S}$ be a functional ws-sTs equipped with a $\omega^{2}$-wqo. Then $\sqsubseteq$ and $\sqsubseteq \mathcal{L}_{0}$ coincide over $\mathcal{S}$.

Example 3. Both wSN (not necessarily affine) and wa are functional by definition. As mentioned in Section 2.2 $\sqsubseteq$ coincides with $\leq$ in the interpretation of the observation function given by $\mathrm{O}(m)=\{i \mid m(i) \neq 0\}$. In the other two cases discussed in Section 2 , the result is more interesting, as $\sqsubseteq$, hence $\sqsubseteq \mathcal{L}_{0}$, is a much coarser relation.

\section{Undecidability of the Spatial-Behavioral Preorder}

The spatial-behavioral preorder $\sqsubseteq$ is in general undecidable in (effective) ws-sTs even if it is the preorder induced by the decidable logic $\mathcal{L}_{0}$. The proof is by reduction from 
the boundedness problem for reset nets (RN) [11]. RN correspond to the subset of affine well-structured nets in which the matrix A, used in the definitions of the functions $f$, contains only the value 0 excluding some value equal to 1 in the main diagonal. This model can be seen as an extension of PN in which transitions can remove all the tokens in some given places. Given a RN and an initial state, the boundedness problem consists of checking whether the set of reachable states is finite. This problem is proved to be undecidable for RN in [11].

Consider a reset net $\Sigma$ with $p$ places and initial marking $m$. It is not restrictive to assume that there is no function $f(x)=A x+B$ defined for $x=0^{p}$. We define an affine wSN $\left[[\Sigma]=\left(\mathbb{N}^{p+4}, F, \preceq\right)\right.$ (where $\leq$ is the usual ordering on naturals extended to vectors) such that there exist two states $s_{1}=0^{p} \cdot(0,0,1,0)$ and $s_{2}=m \cdot(1,0,0,0)$ of $[[\Sigma]]$ such that $s_{1} \sqsubseteq s_{2}$ iff $\Sigma$ is unbounded. In the constructed wsN we will consider a very simple atomic predicate notEmpty and the interpretation exploiting the following labelling function $l:\{1, \ldots, p+4\} \rightarrow \mathcal{P}(\mathrm{At})$, such that $l(i)=\{$ notEmpty $\}$, for $i=p+2$ and $i=p+4$, and $l(i)=\emptyset$, otherwise. The idea that we follow in the definition of $[[\Sigma]]$ is as follows:

- The state $s_{1}=0^{p} \cdot(0,0,1,0)$ can give rise to computations of length $n$, for every $n$, that first increment by one the value in position $p+3$ until the value $n$ is reached, and then such value is moved in position $p+4$ yielding the state $0^{p} \cdot(0,0,0, n)$.

- The state $s_{2}=m \cdot(1,0,0,0)$ can mimic every computation $m \rightarrow^{*} m^{\prime}$ in $\Sigma$. Every time a transition is performed, the value in position $p+1$ is increased by one, thus the state $m^{\prime} \cdot(k, 0,0,0)$ is reached assuming that $k-1$ steps have been simulated. An additional transition can move all the tokens in the marking $m^{\prime}$ in position $p+2$ and set to 0 the value in position $p+1$, thus yielding the state $0^{p} \cdot\left(0, \# m^{\prime}, 0,0\right)$ where $\# m^{\prime}$ denotes the total number of tokens in the marking $m^{\prime}$.

The final states $0^{p} \cdot(0,0,0, n)$, for every $n$, and $0^{p} \cdot\left(0, \# m^{\prime}, 0,0\right)$, for every marking $m^{\prime}$ reachable in $\Sigma$, of the computations starting from $s_{1}$ and $s_{2}$ are related by the atomic predicate notEmpty (able to observe the values in positions $p+2$ and $p+4$ ). We will prove that this relationship guarantees that $s_{1} \sqsubseteq s_{2}$ iff $\Sigma$ is unbounded.

We formally introduce $[[\Sigma]]$ defining its set $F$ that contains the following functions:

- $f_{1}$ is a function that increments by one the value in position $p+3$ if it is greater than 1. Namely, $f_{1}(x)=A x+B$, defined only if $x(p+3)>0$, where $A$ is the identity matrix and $B=0^{p} \cdot(0,0,1,0)$.

- $f_{2}$ is a function that moves the value in position $p+3$ to position $p+4$. Namely, $f_{2}(x)=A x+B$ where $A(i, j)=0$ for every $i$ and $j$, excluding $A(p+4, p+3)=1$, and $B=0^{p+4}$.

- A set of functions that simulate the computation steps of $\Sigma$ (when the value in position $p+1$ is greater than 1) and increment the value in position $p+1$. Namely, for every function $f(x)=A x+B$ in the definition of $\Sigma$, we consider a function $f^{\prime}\left(x^{\prime}\right)=A^{\prime} x^{\prime}+B^{\prime}$, defined only if $x^{\prime}=x \cdot(1,0,0,0)$ and $f$ is defined for $x$, where $A^{\prime}(i, j)=A(i, j)$ for every $1 \leq i, j \leq p$ and $A^{\prime}(i, j)=0$ for every $p+1 \leq i, j \leq p+4$, and $B^{\prime}=B \cdot(1,0,0,0)$.

- $f_{3}$ is a function that moves all the tokens in the marking reached after the simulated computation of $\Sigma$ in position $p+2$. Namely, $f_{3}(x)=A x+B$ where $A(i, j)=0$ for every $i$ and $j$, excluding $A(p+2, j)=1$ for $1 \leq j \leq p$, and $B=0^{p+4}$. 
- $f_{4}$ is a function that permits to restart the simulation of $\Sigma$ if the value in position $p+1$ is not 0 . Namely, $f_{4}(x)=A x+B$, defined only if $x(p+1)>0$, where $A(i, j)=0$ for every $i$ and $j$, and $B=m \cdot(1,0,0,0)$.

We now prove the correctness of the reduction.

Theorem 4. Let $\Sigma$ be a reset net with inital marking $m$. Consider the affine wsN system $\llbracket \Sigma]$, and the states $s_{1}=0^{p} \cdot(0,0,1,0)$ and $s_{2}=m \cdot(1,0,0,0)$. We have that $s_{1} \sqsubseteq s_{2}$ iff $\Sigma$ is unbounded.

Proof: We first consider the if part. Assume that $s_{1} \sqsubseteq s_{2}$. Given a natural number $n$, we will prove that $\Sigma$ has a computation starting from $m$ and leading to a marking with at least $n$ tokens, from which the unboundedness of $\Sigma$ follows. Consider a computation of $\llbracket \Sigma \rrbracket$ of length $n$ starting from $s_{1}$ and leading to $0^{p} \cdot(0,0,0, n)$. As $s_{1} \sqsubseteq s_{2}$ we have that $\llbracket \Sigma \rrbracket$ has a computation starting from $s_{2}$ and leading to a state $0^{p} \cdot\left(0, n^{\prime}, 0,0\right)$ with $n^{\prime} \geq n$. As the computations starting from the state $s_{2}$ mimic computations of $\Sigma$ before moving all the tokens in the reached marking in position $p+2$, we have that also in $\Sigma$ there is a computation from $m$ leading to a marking with $n^{\prime}$ tokens.

We now consider the only-if part. Assume that $\Sigma$ is unbounded. In this case we have that $\Sigma$ has an infinite computation $m=m_{1} \rightarrow m_{2} \rightarrow \cdots \rightarrow m_{i} \rightarrow \cdots$ with the following property: there exists an infinite increasing sequence of indexes $1=l_{1}, l_{2}, \ldots, l_{j}, \ldots$ such that if $j<j^{\prime}$ then $\# m_{l_{j}}<\# m_{l_{j^{\prime}}}$. We now prove that $s_{1} \sqsubseteq s_{2}$ showing the existence of a spatial-behavioral simulation $\mathcal{R}$ between states of $[[\Sigma]]$ such that $\left(s_{1}, s_{2}\right) \in \mathcal{R}$. Let $\mathcal{R}$ be the relation including the following pairs:

1. $\left(0^{p} \cdot(0,0, k, 0), m_{l_{j}} \cdot\left(k^{\prime}, 0,0,0\right)\right) \in \mathcal{R}$ for every $0<k \leq k^{\prime} \leq l_{j}$, where $m_{l_{j}}$ is taken from the computation of $\Sigma, m_{l_{1}} \rightarrow^{+} m_{l_{2}} \rightarrow^{+} \ldots \rightarrow^{+} m_{l_{j}} \rightarrow^{+} \cdots$, with \#m $m_{l_{j}}<\# m_{l_{j^{\prime}}}$ for every $j<j^{\prime}$, described above,

2. $\left(0^{p} \cdot(0,0,0, k), 0^{p} \cdot\left(0, k^{\prime}, 0,0\right)\right) \in \mathcal{R}$ for every $k \leq k^{\prime}$,

3. $\left(0^{p+4}, 0^{p+4}\right) \in \mathcal{R}$,

4. $\left(0^{p} \cdot(0,0, k, 0), 0^{p} \cdot(k, 0,0,0)\right) \in \mathcal{R}$ for every $k>0$.

The relation $\mathcal{R}$ is a spatial-behavioural simulation as it satisfies the three conditions in the Definition 2. There are only two non-trivial conditions to be checked. The first one is that the pairs $\left(0^{p} \cdot(0,0, k, 0), 0^{p} \cdot(k, 0,0,0)\right)$ satisfy condition $(1)$. This holds because of the function $f_{4}$ that allows the second state $0^{p} \cdot(k, 0,0,0)$ to restart the simulation of the computation $m_{1} \rightarrow m_{2} \rightarrow \cdots \rightarrow m_{i} \rightarrow \cdots$. The second one is that the pairs $\left(0^{p} \cdot(0,0, k, 0), m_{l_{j}} \cdot\left(k^{\prime}, 0,0,0\right)\right)$ satisfy condition $(2)$. In this case we observe that $0^{p} \cdot(0,0, k, 0)=t_{1}+t_{2}$ iff $t_{1}=0^{p} \cdot\left(0,0, k_{1}, 0\right)$ and $t_{2}=0^{p} \cdot\left(0,0, k_{2}, 0\right)$ with $k=k_{1}+k_{2}$. If $k_{1}=k$ and $k_{2}=0$ we simply observe that $m_{l_{j}} \cdot\left(k^{\prime}, 0,0,0\right)=m_{l_{j}} \cdot\left(k^{\prime}, 0,0,0\right)+0^{p+4}$. If $k_{1}<k$ we observe that $m_{l_{j}} \cdot\left(k^{\prime}, 0,0,0\right)=0^{p} \cdot\left(k_{1}, 0,0,0\right)+m_{l_{j}} \cdot\left(k^{\prime}-k_{1}, 0,0,0\right)$ and that $\left(0^{p} \cdot\left(0,0, k_{1}, 0\right), 0^{p} \cdot\left(k_{1}, 0,0,0\right)\right) \in \mathcal{R}$ and $\left(0^{p} \cdot\left(0,0, k_{2}, 0\right), m_{l_{j}} \cdot\left(k^{\prime}-k_{1}, 0,0,0\right)\right) \in \mathcal{R}$. We complete the proof observing that $\left(s_{1}, s_{2}\right) \in \mathcal{R}$ as $\left(0^{p} \cdot(0,0,1,0), m \cdot(1,0,0,0)\right) \in \mathcal{R}$ due to the first item of the definition of $\mathcal{R}$ and because $m=m_{1}$.

In the light of this theorem and knowing from [11] that the boundedness problem in reset nets is undecidable, we can conclude that the spatial-behavioral simulation $\sqsubseteq$ is undecidable for affine wSN. 


\section{Conclusion}

We have investigated connections between spatial logic and simulation relations, and related decidability issues, in a general setting of spatial transition systems. One of our results states the coincidence, under certain assumptions, of the logical preorder and of the largest sBs. In the setting of the Ambient Calculus and Ambient Logic, similar results have been achieved by Sangiorgi \& al. in [20, 24]. On the one hand, the clauses of our sBs are reminiscent of their intensional bisimilarity. On the other hand, their completeness proof relies on techniques very different from ours; in particular, the presence in the logic of an adjunct of $\mid$ helps them in defining characteristic formulae for processes in a syntax-driven way, which can have no counterpart in our framework. (Un)decidability of the Ambient Logic is also investigated in [20]. Caires and Lozes study the power of the adjunct in [8]; they too offer Hennessy-Milner theorems based on characteristic formulae and undecidability results relatively to a small fragment of ccs, but they consider a strong, rather than a weak next-step modality as we do. In the setting of the pi-calculus, Caires [5] offers a Hennessy-Milner theorem and decidability results for a Spatial Logic without adjunct, again considering strong modalities and relying on characteristic formulae.

The reader may observe that, while decidability of the monotone spatial logic $\mathcal{L}_{0}$ holds of course for all instances of the framework, the undecidability results are proved in the setting of Affine Well-Structured Nets (wSN). As for future work, we plan to investigate this issue further, so as to obtain new decidability results, or even abstract undecidability results that holds for a whole sub-class of models. Concerning the first direction, we observe that the decidability of sBs seems connected to the problem of effective computation of the finite clover set (see [15]) for $\downarrow$ Post* $(s)$, and this turns out to be effective if we move from reset/transfer Petri nets to Petri nets. Another issue left open by our study is, in the case of wA, the characterization of semirings for which a pred-basis is effectively computable.

Acknowledgment. We thank the anonymous referees for their useful comments on the submitted version of this paper. The second author wishes to thank Jean GoubaultLarrecq for stimulating discussions on complete wsTs.

\section{References}

1. Acciai, L., Boreale, M., Zavattaro, G.: On the relationship between spatial logics and behavioral simulations. Tech. rep. (2010), http://rap.dsi.unifi.it/ acciai

2. Acciai, L., Boreale, M.: Deciding safety properties in infinite-state pi-calculus via behavioural types. In: Albers, S., Marchetti-Spaccamela, A., Matias, Y., Nikoletseas, S., Thomas, W. (eds.) ICALP 2009. LNCS, vol. 5556, pp. 31-42. Springer, Heidelberg (2009)

3. Baccelli, F., Cohen, G., Olsder, G.J., Quadrat, J.P.: Synchronization and linearity. Wiley, Chichester (1992)

4. Busi, N., Gabbrielli, M., Zavattaro, G.: Comparing Recursion, Replication, and Iteration in Process Calculi. In: Díaz, J., Karhumäki, J., Lepistö, A., Sannella, D. (eds.) ICALP 2004. LNCS, vol. 3142, pp. 307-319. Springer, Heidelberg (2004) 
5. Caires, L.: Behavioural and Spatial Observations in a Logic for the pi-Calculus. In: Walukiewicz, I. (ed.) FOSSACS 2004. LNCS, vol. 2987, pp. 72-89. Springer, Heidelberg (2004)

6. Caires, L., Cardelli, L.: A spatial logic for concurrency (part II). Theor. Comput. Sci. 322(3), 517-565 (2004)

7. Caires, L., Cardelli, L.: A spatial logic for concurrency (part I). Inf. Comput. 186(2), 194 235 (2003)

8. Caires, L., Lozes, E.: Elimination of Quantifiers and Undecidability in Spatial Logics for Concurrency. In: Gardner, P., Yoshida, N. (eds.) CONCUR 2004. LNCS, vol. 3170, pp. 240 257. Springer, Heidelberg (2004)

9. Cardelli, L., Gordon, A.D.: Anytime, Anywhere: Modal Logics for Mobile Ambients. In: Proc. of POPL, pp. 365-377 (2000)

10. Dickson, L.E.: Finiteness of the odd perfect and primitive abundant numbers with $r$ distinct prime factors. Amer. Journal Math 35, 413-422 (1913)

11. Dufourd, E.C., Finkel, A., Schnöebelen, P.: Reset Nets Between Decidability and Undecidability. In: Larsen, K.G., Skyum, S., Winskel, G. (eds.) ICALP 1998. LNCS, vol. 1443, pp. 103-115. Springer, Heidelberg (1998)

12. Esparza, J., Finkel, A., Meyr, R.: On the Verification of Broadcast Protocols. In: Proc. of LICS, pp. 352-359 (1999)

13. Esparza, J.: On the Decidability of Model Checking for Several $\mu$-calculi and Petri Nets. In: Tison, S. (ed.) CAAP 1994. LNCS, vol. 787, pp. 115-129. Springer, Heidelberg (1994)

14. Finkel, A., Goubault-Larrecq, J.: Forward Analysis for WSTS, Part I: Completions. In: Proc. of STACS, Dagstuhl Seminar Proceedings 09001, pp. 433-444 (2009)

15. Finkel, A., Goubault-Larrecq, J.: Forward Analysis for WSTS, Part II: Complete WSTS. In: Albers, S., Marchetti-Spaccamela, A., Matias, Y., Nikoletseas, S., Thomas, W. (eds.) ICALP 2009. LNCS, vol. 5556, pp. 188-199. Springer, Heidelberg (2009)

16. Finkel, A., McKenzie, P., Picaronny, C.: A Well-Structured Framework for Analysing Petri Net Extensions. Information and Computation 195(1-2), 1-29 (2004)

17. Finkel, A., Schnöebelen, P.: Well-Structured Transition Systems Everywhere! Theoretical Computer Science 256(1-2), 63-92 (2001)

18. Hack, M.H.T.: Decidability questions for Petri nets. Ph.D Thesis. MIT (1976)

19. Hennessy, M., Milner, R.: On Observing Nondeterminism and Concurrency. In: de Bakker, J.W., van Leeuwen, J. (eds.) ICALP 1980. LNCS, vol. 85, pp. 299-309. Springer, Heidelberg (1980)

20. Hirschkoff, D., Lozes, E., Sangiorgi, D.: Separability, Expressiveness, and Decidability in the Ambient Logic. In: Proc. of LICS, pp. 423-432 (2002)

21. Kuich, W., Salomaa, A.: Semirings, Automata, Languages. Monographs in Theoretical Computer Science, EATCS Series, vol. 5. Springer, Heidelberg (1986)

22. Milner, R.: Communication and concurrency. Prentice-Hall, Englewood Cliffs (1989)

23. Minsky, M.: Computation: Finite and Infinite Machines, 1st edn. Prentice-Hall, Inc., Englewood Cliffs (1967)

24. Sangiorgi, D.: Extensionality and Intensionality of the Ambient Logics. In: Proc. of POPL, pp. 4-13 (2001)

25. Simon, I.: Limited subset of a Free Monoid. In: Proc. of FOCS, pp. 143-150 (1978)

26. Valk, R., Jantzen, M.: The residue of vector sets with applications to decidability problems in Petri nets. Acta Informatica 21, 643-674 (1985) 